\title{
Start Rule
}

National Cancer Institute

\section{Source}

National Cancer Institute. Start Rule. NCI Thesaurus. Code C82566.

An axiom that indicates the start of an event, visit or study element. 